Project No. 09-783

\title{
Improvements to Nuclear Data and Its Uncertainties by Theoretical Modeling
}

\section{Insert Fuel Cycle/Reactor Concepts}

Dr. Yaron Danon

Rensselaer Polytechnic Institute

In collaboration with: University of Tennessee, Knoxville

University of New Mexico Los Alamos National Laboratory

Tansel Selekler, Federal POC Robert Hill, Technical POC 


\title{
IMPROVEMENTS OF Nuclear Data AND ITS UnCeRTAINTIES BY THeORETical Modeling
}

FinAl RePort of AFCI-NEUP ProJect \# 09-247

\author{
Y. Danon
}

Gaerttner LINAC Laboratory, Rensselaer Polytechnic Institute, NY 12180, USA

W. Nazarewicz

Department of Physics and Astronomy, University of Tennessee

Knoxville, TN 37996, USA

A.K. Prinja

Chemical and Nuclear Engineering Department, University of New Mexico, USA

P. Talou*

Nuclear Theory Group, Los Alamos National Laboratory, Los Alamos, NM, USA

January 29, 2013

\section{Executive Summary}

This document reports on the results obtained during the 3-year AFCI-NEUP project \#09-247, which aimed at improving the modeling and evaluation of nuclear data and their uncertainties for use in advanced nuclear energy transport simulations. Several important results and outcomes of this project can be reported:

1) Advanced calculations of prompt fission neutron and $\gamma$ ray spectra, multiplicities, distributions and correlations, on a fission even-by-event basis;

2) Fully microscopic calculations of fission using UNEDF1;

3) Consistent evaluation of prompt fission neutron spectra and their associated covariance matrices for suites of $\mathrm{Pu}$ and $\mathrm{U}$ isotopes;

4) New and very efficient approach to propagating uncertainties in transport simulations.

Each one of these results is opening new venues for computing physical quantities of relevance to the nuclear fuel cycle: prompt fission neutron and $\gamma$-ray correlations are being proposed for advanced detector simulations and for inclusion in the MCNP6 transport code; consistent fission cross section calculations across suites of isotopes using fully microscopic input data; cross-isotope evaluations and uncertainty quantifications are being proposed for the U.S. ENDFB-VII library for the first time; new covariance matrix capabilities in transport simulations are being developed. These important results have been reported in multiple refereed publications, international conferences and meetings.

\footnotetext{
*Email: talou@lanl.gov
} 


\section{Introduction}

Several significant gaps in evaluated nuclear data libraries, important for nuclear energy applications, had been identified. This project proposed to make significant contributions to address three of these issues. Here, we report on the results of this 3-year project that involved three U.S. Universities in collaboration with one DOE National Laboratory. The main contributors to this work are listed below:

- Rensselaer Polytechnic Institute, NY: Y. Danon (PI), B. Becker (postdoc), E. Blain (PhD)

- University of Tennessee, TN: W. Nazarewicz, J. McDonnell (PhD), N. Nikolov (PhD)

- University of New Mexico, NM: A.K. Prinja, M.E. Rising (PhD)

- Los Alamos National Laboratory, NM: P. Talou

B. Becker has now moved to a postdoctoral position at the IRMM, Geel, Belgium; J. McDonnell completed his $\mathrm{PhD}$ at UTK successfully, and is now in a postdoctoral position in the Nuclear Theory Group at LLNL; M.E. Rising completed his PhD at UNM successfully and is now employed as a postdoc at LANL.

The three important issues that have been addressed in this project are the following:

- Prompt fission neutrons and $\gamma$ Rays

- Consistent input for fission cross section modeling

- Uncertainty Quantification (UQ) tools

The following sections summarize our results in each of these three items.

\section{Prompt Fission Neutrons and $\gamma$ Rays}

Most modern evaluations of prompt fission neutrons rely on a modified version of the Madland-Nix or Los Alamos model [1], which provides a relatively straightforward way of calculating an average prompt fission neutron spectrum (PFNS) and average prompt fission neutron multiplicity (PFNM), although the PFNM is more often obtained from an analysis of experimental data when available. This model was developed by D.G. Madland and J.R. Nix at LANL in the 1980s, and has been very successful in predicting PFNS for incident neutrons from thermal up to $20 \mathrm{MeV}$, and for a wide range of actinide targets. Only a few model input parameters are needed to compute the average prompt neutron spectra $\langle\chi\rangle\left(E_{\text {in }}, E_{\text {out }}\right)$ and average prompt neutron multiplicity $\bar{v}\left(E_{\text {in }}\right)$. Those parameters can be fitted to available spectrum measurements, if any. While this model has been very successful, it cannot predict anything beyond those two averaged quantities.

Moving beyond average quantities requires to follow in detail the sequence of successive neutron and photon emissions that lead to the de-excitation of the primary fission fragments into more stable configurations (but before a possible further beta-decay). This is exactly what we have done with two LANL-developed codes, FFD [2, 3, 4] and CGMF [5, 6]. Both codes are Monte Carlo implementations of well-established statistical nuclear reaction theories, which are commonly used in the evaluation of nuclear reaction cross sections that are contained in the ENDF/B library and used in most transport simulations. 
The FFD code describes the neutron evaporation from the fission fragments using the Weisskopf emission theory, similar to what is used on average in the Madland-Nix model. The fission fragment yields as a function of mass, charge and total kinetic energy are sampled to provide the initial conditions for the FFD code. This code performs a Monte Carlo sampling of the initial excitation energies in each light and heavy fragment pairs, and evaporates neutrons following a Weisskopf spectrum at a given temperature. Neutrons are emitted in sequence until the residual excitation energy falls below the neutron separation energy. At that point, all the remaining energy is going to be dissipated through prompt $\gamma$ rays.

Such a detailed approach allows the study of more exclusive data such as the neutron multiplicity distribution $P(v)$, exclusive spectra for a specific multiplicity $\chi\left(E_{\text {out }}\right)_{\mid v=1,2, \ldots}$, neutron-neutron energy and angular correlations, etc.

The CGMF code goes a step further and computes the de-excitation of the fragments by emission of both neutrons and photons, following the Hauser-Feshbach theory that keeps track of all quantum numbers during the decay. While FFD could only compute neutrons, CGMF can compute the same type of data for prompt gamma rays as well. Its implementation is however much slower due to additional requirements of calculating the neutron-gamma competition at every stage of the decay.

In this project, the following milestones have been successfully completed:

- Merging of CGM and FFD to create a new code, CGMF

- Implementation and optimization of CGMF on a parallel machine

- Sensitivity studies of FFD on input parameters, such as fission fragment yields

- Deliver new data on prompt fission neutrons for selected fission reactions

- First ever calculations of prompt fission gamma ray data for selected fission reactions

\subsection{A New Code CGMF}

At the beginning of this project, we had developed two codes: FFD, which treats the decay of primary fission fragments using the Weisskopf theory; and CGM , which is a Monte Carlo implementation of the Hauser-Feshbach theory treating both neutron and gamma-ray emissions in competition. In the course of this project, we successfully developed a new code, CGMF, which represents a merger of the capabilities of both codes. New C++ classes have been coded to extend CGM to the fission problem. Treating properly the competition between prompt neutrons and gamma rays is very CPU-intensive. We developed an MPI version of the CGMF code that can use parallel computers very efficiently.

\subsection{FFD Sensitivity Studies}

We have studied the sensitivity of the results obtained with the FFD code on the choice of the initial fission fragment yield distributions $\mathrm{Y}(\mathrm{A}, \mathrm{Z}, \mathrm{TKE})$. The distributions $\mathrm{Y}(\mathrm{A}, \mathrm{TKE})$ were measured at RPI [7] for ${ }^{252} \mathrm{Cf}$ ( $\mathrm{sf}$ ), $\mathrm{n}+{ }^{239} \mathrm{Pu}$, and $\mathrm{n}+{ }^{235} \mathrm{U}$, for incident neutrons from thermal to $10 \mathrm{keV}$ energies. The lead slowing down spectrometer (LSDS) at RPI was used to measure fission cross-sections and fission fragment yields simultaneously.

In this first year, we partially re-analyzed those experimental yields to fully understand and account for all sources of uncertainties leading to statistical and systematic uncertainties in the measured yields. These uncertainties were then propagated into the FFD code to study their impact on the results of the calculations. 

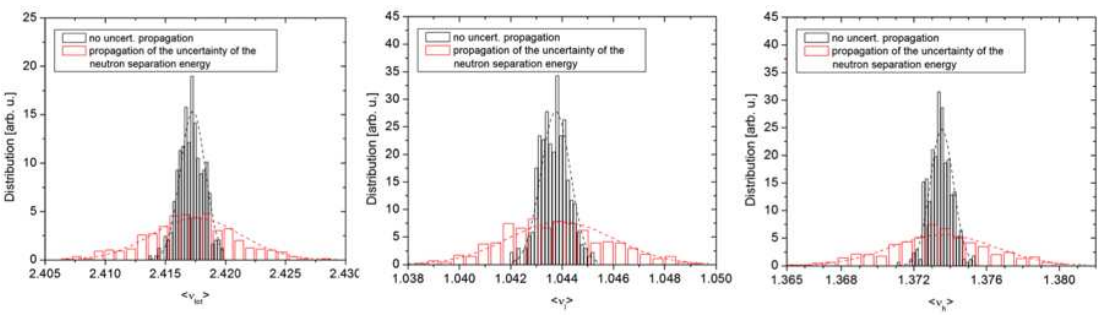

Figure 1: The statistical distributions around the average neutron multiplicities are enlarged when taking into account uncertainties in the model input parameters.

Other uncertainties due to nuclear data parameters used in the FFD code were included in this analysis, such as neutron separation energies, level density parameters, etc. Figure 1 shows the impact of including uncertainties in the neutron separation energies on the final calculated average neutron multiplicities for the light and heavy fragments, as well as the total. Uncertainties propagated to the average spectrum and other quantities calculated with FFD are now being investigated.

This sensitivity work is being pursued further using the CGMF code this time.

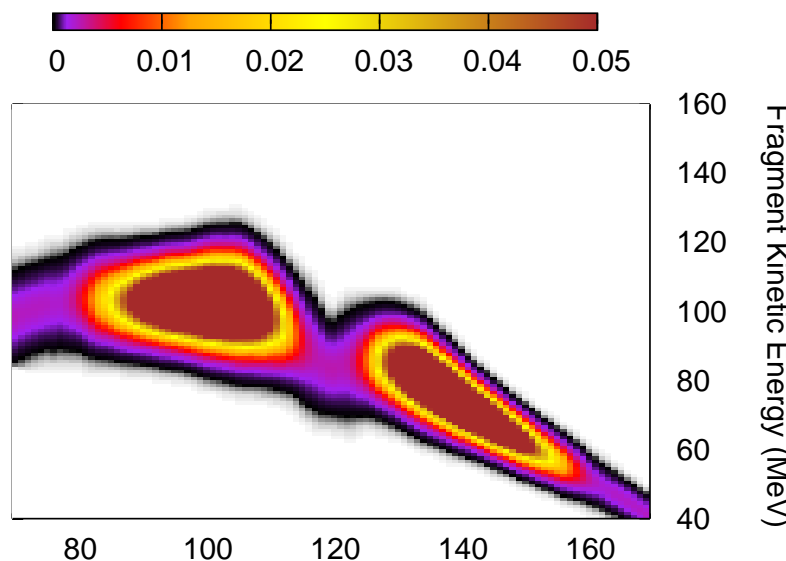

Fragment Mass (amu)

Figure 2: Primary fission fragment yields $\mathrm{Y}(\mathrm{A}, \mathrm{KE})$ for the thermal neutron-induced fission reaction on $\mathrm{Pu}-239$.

\subsection{New Data on Prompt Fission Neutrons}

FFD calculations were performed for the thermal neutron-induced fission on $\mathrm{Pu}-239$ and results were published in Ref. [8]. The primary fission fragment yields vs. mass, charge and kinetic energy were carefully produced using a combination of least-square analysis of experimental data and systematics. Figure 2 shows the fission fragment yields $Y(A, K E)$ as a function of the fragment mass and kinetic energy. 
From the mass, charge and total kinetic energy values from a pair of fragments produced in a single fission event, it is straightforward to extract the total excitation energy (TXE) available for the evaporation of prompt neutrons and gamma rays. However, how TXE is partitioned among the light and heavy fragments remains an open question. In [8], we used a pragmatic solution centered around getting a good fit of the ratio of neutron multiplicities $\bar{v}_{l} / \bar{v}_{h}$ as a function of the heavy fragment mass $A_{h}$.

Once the initial conditions of the primary fission fragments are set, FFD follows the evaporation of neutrons until the excitation energy of the residual nucleus is too low for further neutron emission. The calculated neutron multiplicity was $\bar{v}_{c}=2.871$, in very good agreement with the standard evaluated value of $\bar{v}_{e}=2.8725$. In addition to calculating the average PFNM, one can also calculate its distribution $P(v)$, as shown in Fig. 3.

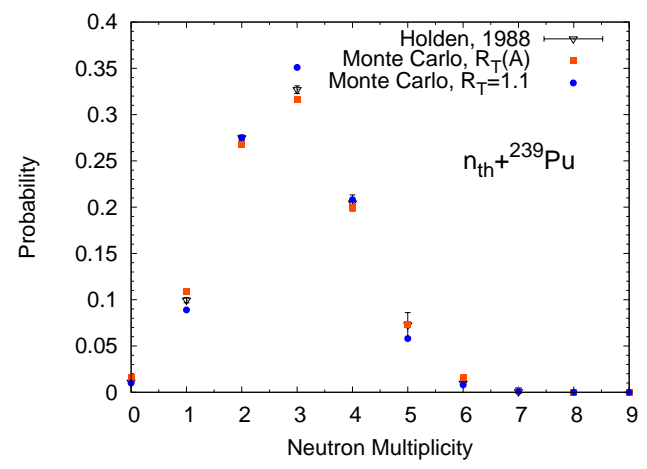

Figure 3: Prompt fission neutron multiplicity distribution for the thermal neutron-induced fission on $\mathrm{Pu}-239$.

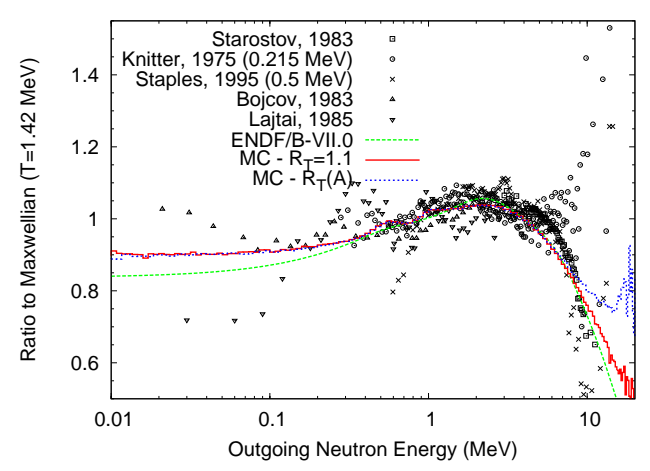

Figure 4: Average prompt fission neutron spectrum calculated for the thermal neutron-induced fission on $\mathrm{Pu}-239$, and compared to experimental data and the ENDF/B-VII.0/1 evaluation.

The calculated average PFNS lies within the evaluated uncertainties of the current ENDF/BVII.0/1 evaluated PFNS, as shown in Fig. 4 (blue and red curves, corresponding to two different assumptions on TXE partitioning).

Many other quantities of interest were reported in [8] and won't be repeated here. Such results are very valuable to better understand the physics post-scission, and to develop advanced simulation tools for transport simulations for nuclear energy and other applications.

\subsection{Calculations of Prompt Fission $\gamma$ Rays}

Evaluated prompt fission $\gamma$-ray data are very scarce and based exclusively on even more limited experimental data sets. Indeed, the Madland-Nix model used very successfully over the years to predict the average prompt fission neutron spectrum and multiplicity says nothing about the emission of prompt $\gamma$ rays, except for the total residual energy that is left after neutron emission.

Thanks to our new CGMF code, a large body of data concerning prompt fission $\gamma$ rays can now be produced. In fact, all quantities that could be inferred for the prompt neutrons from FFD simulations can now be obtained for $\gamma$ rays as well.

Important results have already been obtained [6] for selected fission reactions: $\mathrm{n}_{t h}+{ }^{235} \mathrm{U}, \mathrm{n}_{t h}+{ }^{239} \mathrm{Pu}$, and ${ }^{252} \mathrm{Cf}$ (sf). We present here only a few selected results, mainly taken from our recent publica- 


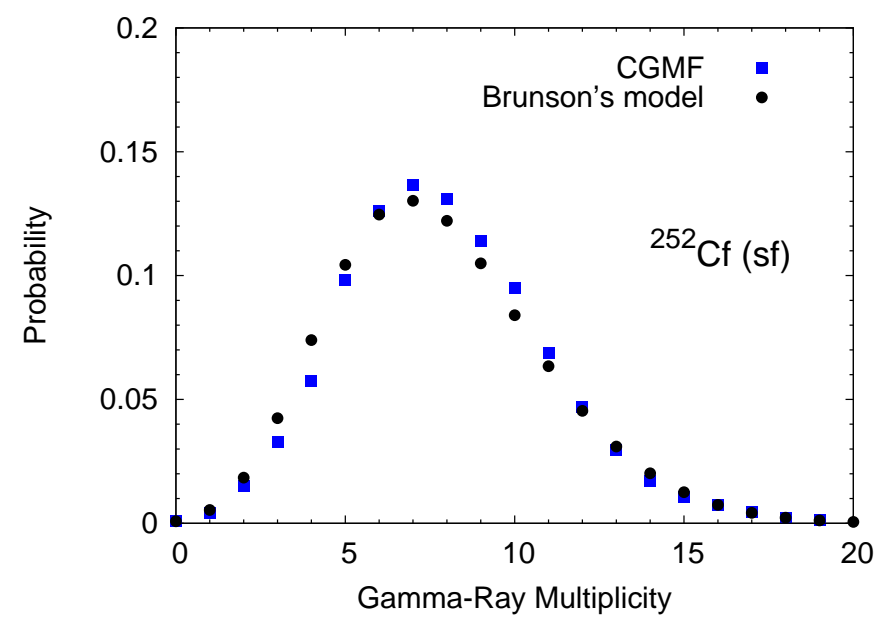

Figure 5: The calculated prompt fission $\gamma$-ray multiplicity distribution $\mathrm{P}\left(\mathrm{N}_{\gamma}\right)$ is compared to calculations from Brunson's double Poisson model.

tion [6].

The calculated prompt fission $\gamma$ multiplicity $\mathrm{P}\left(\mathrm{N}_{\gamma}\right)$ is shown in Fig. 5 and compared to predictions from Brunson's model whose parameters have been fitted to reproduce known experimental data. Our CGMF calculations are in excellent agreement with the Brunson model data.

The average prompt fission $\gamma$-ray spectrum calculated for $n_{t h}+{ }^{235} \mathrm{U}$ is shown in Fig. 6 in comparison with experimental data.. The overall agreement is quite reasonable. Of interest are the fluctuations observed both in the calculations and the experimental data. Those fluctuations are due to specific $\gamma$ transitions in the fission fragments. Low-energy $\gamma$ lines, below $100 \mathrm{keV}$, are difficult to observe, and are also difficult to predict in our calculations. A minimum threshold of $140 \mathrm{keV}$ has been applied to the spectra shown in Fig. 6.

Characteristics of the prompt $\gamma$ rays can also be studied as a function of the fission fragment mass. For instance, the average $\gamma$-ray energy calculated as a function of the fragment mass is shown in Fig. 7. The significant increase observed for masses around 130 is due to the higher temperature predicted for nuclei near shell closures. For those nuclei, the spacing between low-lying levels should be increased, hence increasing the average value for $\epsilon_{\gamma}$.

Since the competition between neutron and $\gamma$ emissions is taken into account explicitly in CGMF simulations, correlations between neutrons and $\gamma$ rays can be inferred. In Fig. 8, the average $\gamma$ multiplicity $\bar{N}_{\gamma}$ and total average energy $\left\langle E_{\gamma}^{\text {tot }}\right\rangle$ are plotted as a function of the average neutron multiplicity $\bar{v}$.

\section{Fission Cross Section Modeling}

A new fission cross-section modeling tool has been developed at LANL, which is based on the original modeling work and code by J.E. Lynn [10, 11]. The R-matrix theory is applied to the fission channel, which uses the concept of fission transition states on top of barrier saddle points. As it is well known, shell-model corrections on top of a collective liquid-drop-type contribution lead to a complicated fission potential energy surface, often simplified as a double-humped barrier. The pres- 


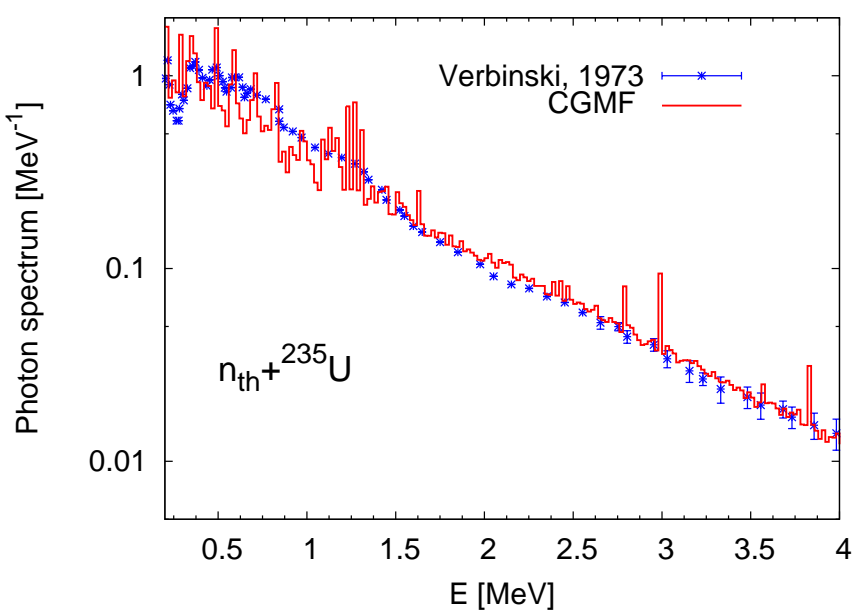

Figure 6: Prompt fission $\gamma$ spectrum calculated with CGMF and compared to experimental data.

ence of this second well leads to several interesting phenomena, and in particular to resonances in the fission probability as a function of the excitation energy. The coupling between class-I and class-II states also lead to significant corrections to the standard Hauser-Feshbach decay probabilities.

Quite a few model parameters enter in the fission cross-section calculations, including the fission barrier characteristics (height, width, inertia). While most of these parameters are constrained by independent measurements, the fission transition states, both discrete and in the continuum, remain largely unknown, while calculated fission cross-sections are very sensitive to the representation of the level densities in the continuum.

\subsection{Microscopic Calculations of Fission Paths}

The work performed at UTK as part of this project aimed at describing fission paths using fully microscopic nuclear energy density functional theory calculations in the Adiabatic Time-Dependent Hartree-Fock-Bogolyubov (ATDHFB) model, and has resulted in several important outcomes. The UNEDF1 energy density functional parametrization was recently developed with fission applications in mind [12]. In a survey of fission barrier properties of even-even actinides, we find that UNEDF1 yields predictions that agree well with experimental values and are on par with, or better than, predictions of other self-consistent or macroscopic-microscopic models. This is illustrated in Fig. 9 that benchmarks UNEDF1 against alternative parametrizations of the energy functional (Skyrme SkM and Gogny D1S14) and the microscopic-macroscopic FRLDM15 models for the inner fission barrier height.

The collective mass was obtained in the case of ${ }^{256} \mathrm{Fm}$, showing strong variations as function of the quadrupole collective coordinate (see Fig. 11). Comparisons were performed with perturbation and cranking approximations in the ATDHFB approach, and with the GOA method. The inertia parameter along the fission path is an important input in fission cross-section calculations. Using these mass parameters, we performed large-scale calculations of spontaneous fission lifetimes for the actinides and superheavy nuclei. Figure 10 shows a very good agreement between experiment and theory for UNEDF1 and SkM* models. 


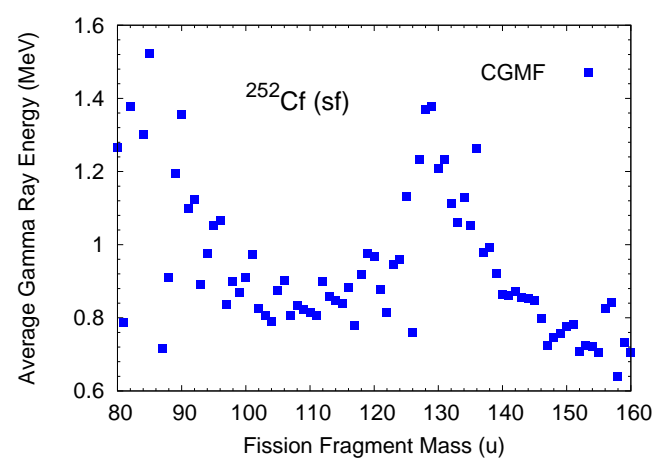

Figure 7: The calculated average $\gamma$-ray energy is plotted as a function of the fission fragment mass.

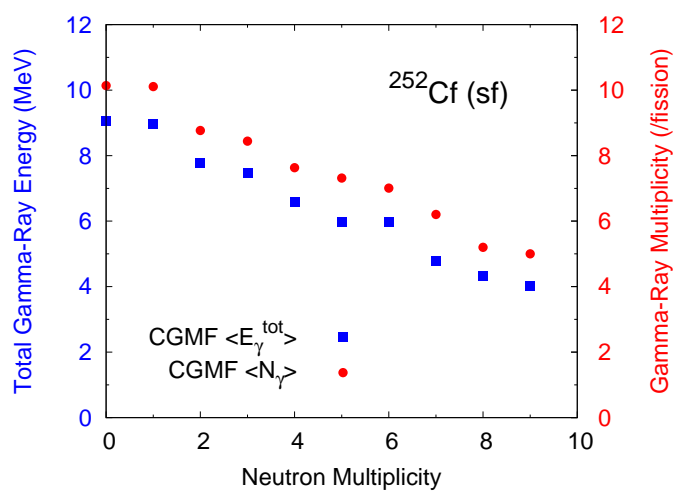

Figure 8: The average $\gamma$ multiplicity $\bar{N}_{\gamma}$ and total average energy $\left\langle E_{\gamma}^{\text {tot }}\right\rangle$ are plotted as a function of the average neutron multiplicity $\bar{v}$. The observed negative slopes are in contradiction with the experimental results of Nifenecker et al. [9].

The potential energy surface calculated in the HFB formalism for ${ }^{232} \mathrm{Th}$ is shown in Fig. 12. At low excitation energies, the fission path exhibits a shallow third, reflection-asymmetric, minimum. This minimum disappears quickly with increasing excitation energy.

Optimal one-dimensional fission paths have been obtained for thorium, fermium as well as plutonium isotopes. Thorium and fermium isotopes are of special interest for fundamental reasons (pronounced third well on the fission path, transition from symmetric to asymmetric fission) while plutonium isotopes are of more direct interest to nuclear energy applications. Of course, all of these calculations are useful to constrain the models, and develop more predictive capabilities.

Finite-temperature Hartree-Fock + BCS calculations were used to compute potential energy surfaces (PES) for many actinides, including ${ }^{240} \mathrm{Pu}$, as a function of temperature. As can be seen in Fig. 13, those calculations show large modifications of the fission barriers for different excitation energies. A proper account of this effect in fission cross section calculations has yet to be implemented explicitly. Also shown on this figure is the fact that symmetric and asymmetric barriers tend to converge at higher temperatures.

Consistent HFB studies of the fission barriers for plutonium isotopes have been performed and delivered to LANL for fission cross section calculations.

\subsection{Fission Cross Section Calculations}

While initial studies have been performed, the complete use of those microscopically-calculated fission barrier parameters in LANL's fission cross section code is beyond the scope of this project. More code developments have to be implemented before such data can be used to their fullest extent. We plan to continue this collaboration work in a separate framework. However, preliminary analyses of those input parameters have already revealed several important improvements that could be included in LANL's calculations. Such improvements should also lead to the development of more predictive capabilities for fission cross section modeling, which could be applied to not so well known cross sections of minor actinides. This is an important goal for advanced reactor simulations. 


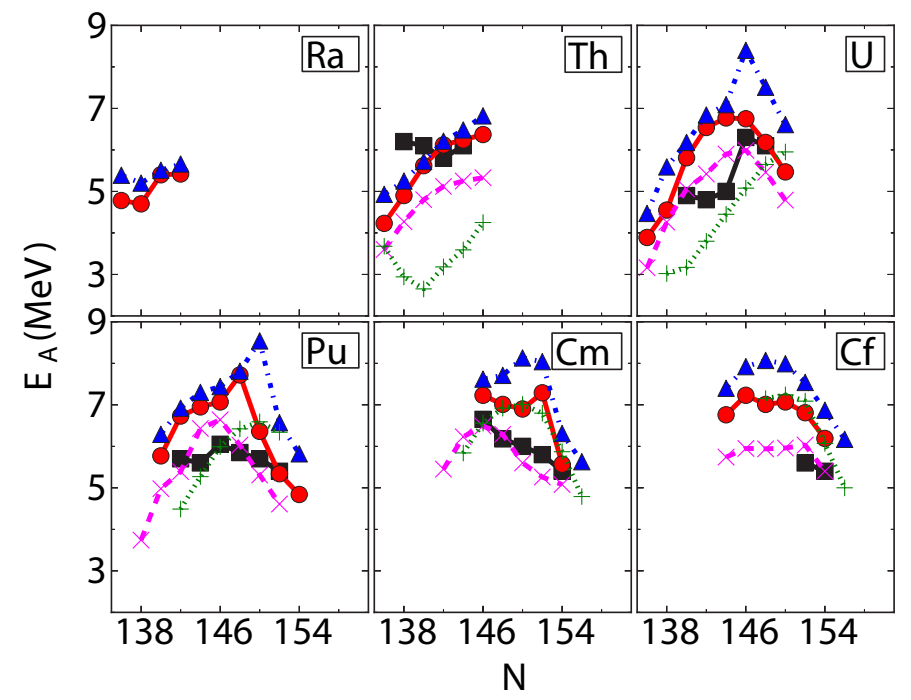

Figure 9: Inner barrier heights of selected even-even actinide nuclei. Experimental data (black lines with square markers) are compared to predictions of UNEDF1 (red lines with circles); SkM (blue lines with triangles); D1S14 (magenta lines with ÔxÕ-es); and FRLDM15 (green lines with plus signs). (From J. McDonnell, N. Schunck, and W. Nazarewicz; Proc. Sanibel Meeting 2012.)

\section{Innovative Data Assimilation Tools}

A third component of this project was to develop new evaluation tools that integrate UQ tools seamlessly in the evaluation process. Because of its importance for many nuclear applications, including GEN-IV reactor simulations, we focused our efforts on the Prompt Fission Neutron Spectrum (PFNS) that describe the energy distribution of the prompt neutrons emitted right around the time of scission, as opposed to $\beta$-delayed neutrons.

At the core of most evaluated PFNS in nuclear data libraries worldwide (ENDF/B, JENDL, JEFF, etc.) lies the Madland-Nix or Los Alamos model [1] for predicting PFNS. This model, developed by D.G. Madland and J.R. Nix at LANL in the early 1980s, has been used very successfully to compute PFNS for a large number of actinides and for a wide range of incident neutron energies, with only a few adjustable parameters.

Recently, LANL developed a modern code, which implements the full set of equations derived in the Los Alamos model. This new code is more robust and much easier to handle than previous LANL capabilities. In this project, UNM and LANL developed a complete package around this code, providing a powerful yet easy-to-use toolkit for the analysis, calculation and evaluation of PFNS and PFNM (multiplicity). This toolkit comprises:

- an extended version of the Los Alamos model equations;

- a Kalman filter for the quantification of uncertainties associated with evaluated PFNS;

- an experimental module to analyze experimental PFNS data and produce realistic experimental covariance matrices;

- an implementation of LA model input parameter systematics; 


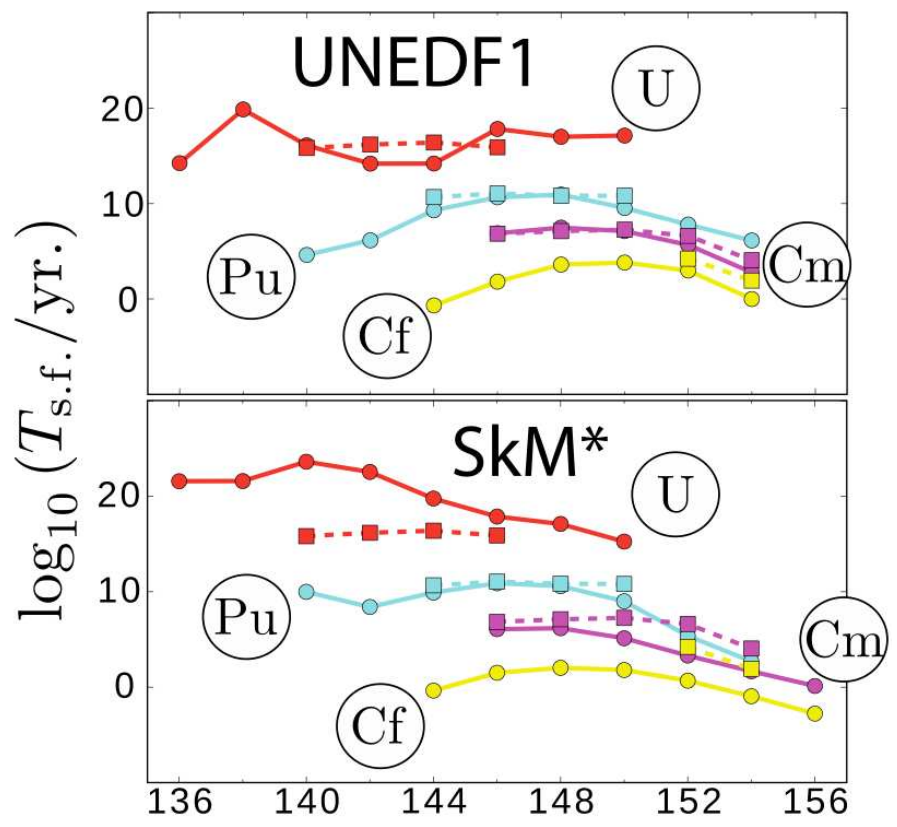

Figure 10: Spontaneous fission half-lives for U, Pu, Cm, and Cf calculated with UNEDF1 (top) and $\mathrm{SkM}^{*}$ (bottom) models and compared to the data. (J. McDonnell, Thesis; in preparation.)

- an ENDF processing package.

\subsection{The Los Alamos model}

We have expanded our initial PFNS code that implements the Los Alamos model [1].

- In its original version, the main result of the LA model concerns the average PFNS in the laboratory system, which can be expressed as

$$
N(E)=\frac{1}{2 \sqrt{E_{f}} T_{m}^{2}} \int_{\left(\sqrt{E}-\sqrt{E_{f}}\right)^{2}}^{\left(\sqrt{E}+\sqrt{E_{f}}\right)^{2}} d \epsilon \sigma_{c}(\epsilon) \sqrt{\epsilon} \int_{0}^{T_{m}} d T k(T) \exp (-\epsilon / k T)
$$

where $k(T)$ is a temperature-dependent normalization factor. This expression is obtained by assuming that the neutrons are emitted from the two fully accelerated fission fragments, whose initial temperatures follow a triangular distribution with a maximum value of $T_{m}$. The energy-dependent term $\sigma_{c}(\epsilon)$ is the cross section for the inverse compound nucleus formation process. The neutrons are assumed to be emitted isotropically in the center-of-mass reference frame of the moving fragments, following the Weisskopf expression

$$
\phi(\epsilon)=\frac{\epsilon}{T^{2}} \exp (-\epsilon / T)
$$

The most relevant input parameters for the Los Alamos model are the average energy release $\left\langle\mathrm{E}_{r}\right\rangle$, the average total kinetic energy $\langle\mathrm{TKE}\rangle$, and the average level density parameter $\langle a\rangle$. Those three parameters define the average temperature of the PFNS, and therefore its hardness or softness. 


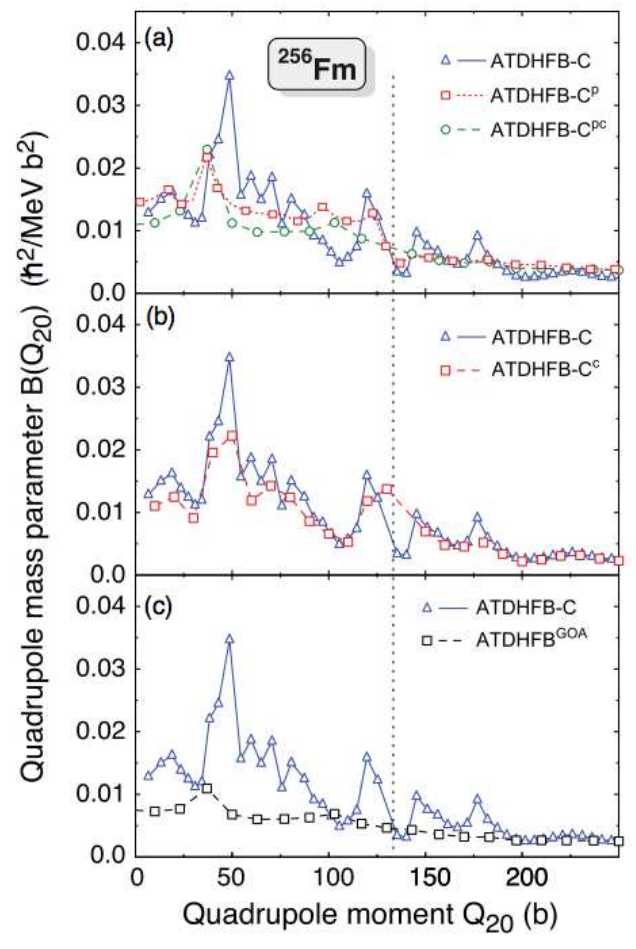

Figure 11: The quadrupole mass parameter is shown as a function of the quadrupole moment $Q_{20}$ along the static fission path of ${ }^{256} \mathrm{Fm}$ calculated in the $\mathrm{SkM}^{*}+\mathrm{HFB}$ formalism [taken from Ref. [13]].

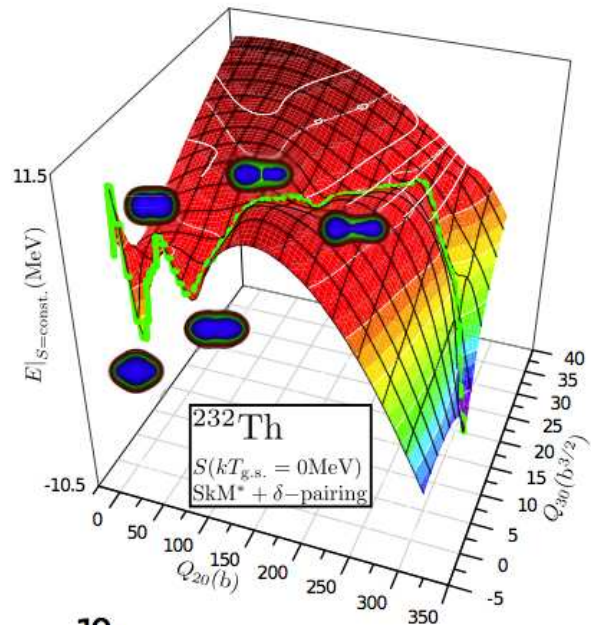

Figure 12: Hartree-Fock-Bogolyubov calculations of the potential energy surface for ${ }^{232} \mathrm{Th}$, showing a third minimum (relectionasymmetric) on the fission path, which eventually disappears with increasing excitation energy.

In its original version again, the final average spectrum is obtained by simply averaging the spectrum of neutrons emitted by the light fragment and the spectrum of its heavy partner. In other words, it is assumed that the same number of neutrons is emitted from the light as well as heavy fragment. Also, it was originally assumed that the temperature $T_{m}$ is identical in the light and heavy fragments.

As part of this work, we have extended the LA model in several ways. First, the temperature in both fragments does not have to be the same, as most of the excitation energy shared between them at scission is stored in collective degrees of freedom, in particular, their deformation, which cannot be shared through thermal exchanges. By the time the fragments start evaporating neutrons, which is assumed to happen once the fragments are fully accelerated, their temperatures can be quite different. On average, it is observed that $\left\langle T_{L}\right\rangle>\left\langle T_{H}\right\rangle$.

The second assumption to be removed is that both fragments emit the same number of neutrons. On the contrary, a large body of experimental data shows that, on average, more neutrons are emitted from the light than from the heavy fragments. So the simple averaging of both spectra can be revised 


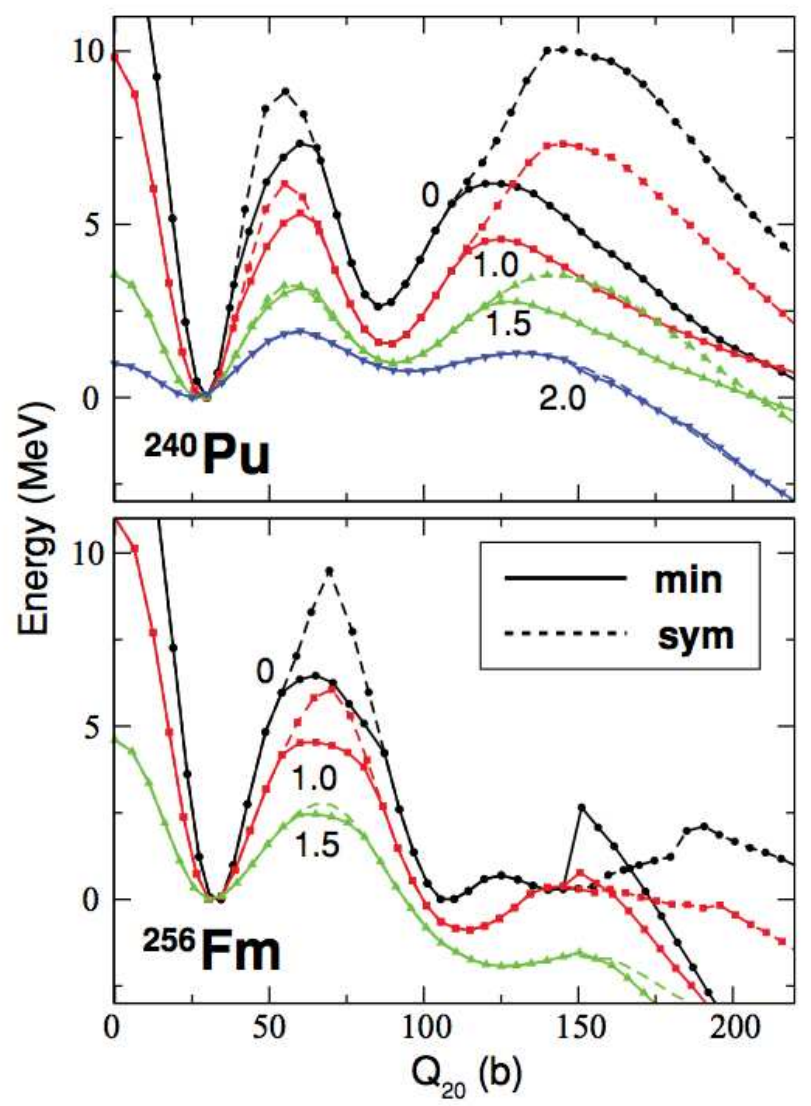

Figure 13: Fission pathways of ${ }^{240} \mathrm{Pu}$ (top) and ${ }^{256} \mathrm{Fm}$ (bottom) calculated as a function of the quadrupole moment $Q_{20}$ for different ground-state temperatures $k T_{\text {g.s. }}$ [taken from Ref. [14]].

as

$$
N(E)=\frac{1}{\bar{v}_{L}+\bar{v}_{H}}\left[\bar{v}_{L} N_{L}(E)+\bar{v}_{H} N_{H}(E)\right]
$$

Finally, it is predicted that neutrons emitted from fragments with large angular momentum will not be isotropic in the center-of-mass of the fragments. A simple extension of Eq. (1) can be derived

$$
\begin{aligned}
N(E)= & \frac{1}{2 \sqrt{E_{f}} T_{m}^{2}} \frac{1}{1+b / 3} \int_{\left(\sqrt{E}-\sqrt{E_{f}}\right)^{2}}^{\left(\sqrt{E}+\sqrt{E_{f}}\right)^{2}} \sigma_{c}(\epsilon) \sqrt{\epsilon}\left(1+b \frac{\left(E-\epsilon-E_{f}\right)^{2}}{4 \epsilon E_{f}}\right) d \epsilon \\
& \times \int_{0}^{T_{m}} k(T) T \exp (-\epsilon / T) d T,
\end{aligned}
$$

where $b$ is the so-called anisotropy parameter. 


\subsection{Los Alamos Model Input Parameter Systematics}

Over the years, Tudora [15] has performed systematic studies of PFNS using the LA model, inferring a set of model input parameters that best fit the data. From this work, she has derived a set of functional forms for the LA model input parameters across suites of isotopes $(\mathrm{U}, \mathrm{Pu}, \mathrm{Np}, \mathrm{Am}$, $\mathrm{Cm})$. We will not repeat those expressions here, but simply state that we have implemented them in our PFNS code package, and used them as prior parameters in our Bayesian statistical analysis of experimental data and model calculations. Because those systematics were developed across suites of isotopes, our evaluation work now links PFNS for different isotopes and hence creates crossisotope correlations in the final evaluated results. To our knowledge, this is the first time, such cross-isotope correlations are evaluated from both model and experimental data. As an example, the average energy release is shown in Fig. 14 as a function of the fissility parameter.

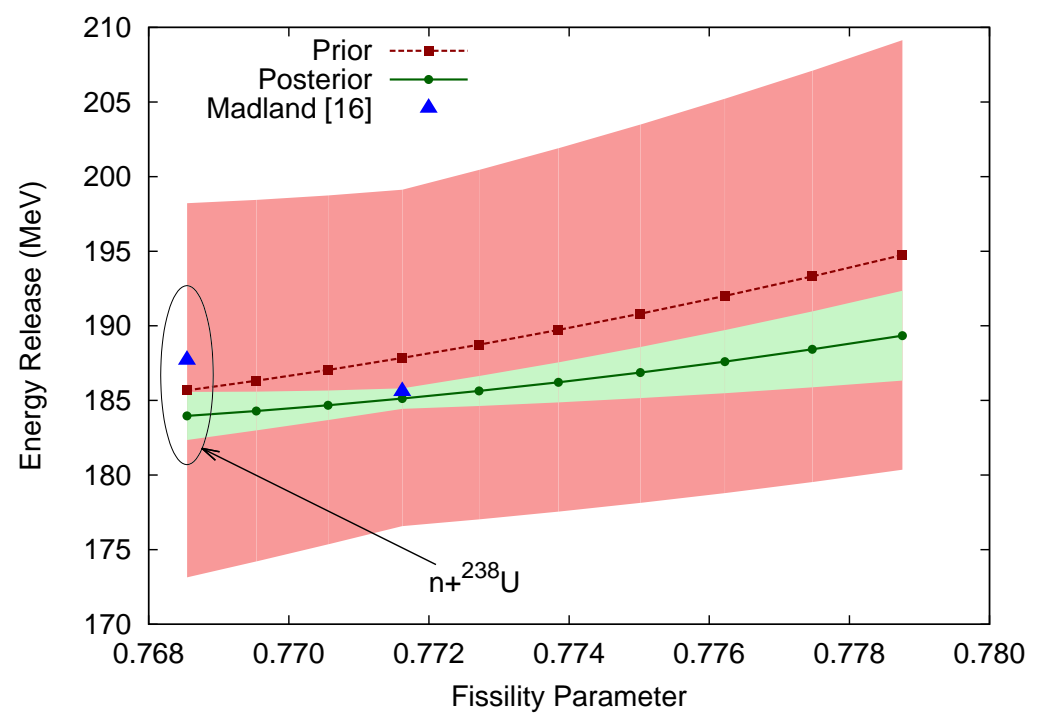

Figure 14: The average energy release parameter entering in Los Alamos calculations is plotted as a function of the fissility parameter. A consistent evaluation of this parameter across a suite of isotopes lead to cross-isotope correlations in the final evaluated PFNS and associated covariance matrices.

\subsection{Experimental Module}

We have developed a new experimental module that analyzes experimental PFNS data sets, produce associated covariance matrices, and prepare those data for inclusion in our Bayesian statistical analysis. PFNS data are different from cross section data in the sense that they always have to be normalized to theoretical calculations before being used in an evaluation procedure. The reason is that PFNS data are always partial: they only correspond to a measured spectrum between a low and high outgoing energy. Since a PFNS is a probability distribution, it is normalized to unity from 0 to infinity in energy. Also, in many instances, experimental PFNS data were taken as a ratio to the standard Cf-252 (sf) PFNS. This module is very versatile and will process most experimental data sets automatically, according to experimental details. 
In addition, a significant effort has been made to produce more realistic experimental covariance matrices than in the past. This is an often neglected part of the evaluation procedure, albeit very important. It is indeed difficult to estimate systematic uncertainties for a given experiment, and even more difficult to address cross-experiment correlations. The latter was not treated in this work. However, many short-, medium- and long-range correlations were estimated for PFNS data used in our evaluation work.

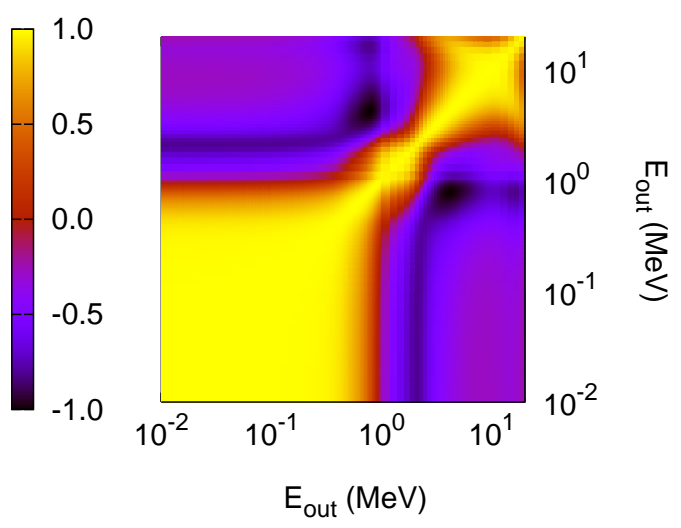

Figure 15: The U-233 PFNS correlation matrix obtained from a complete evaluation using model calculations and experimental data.

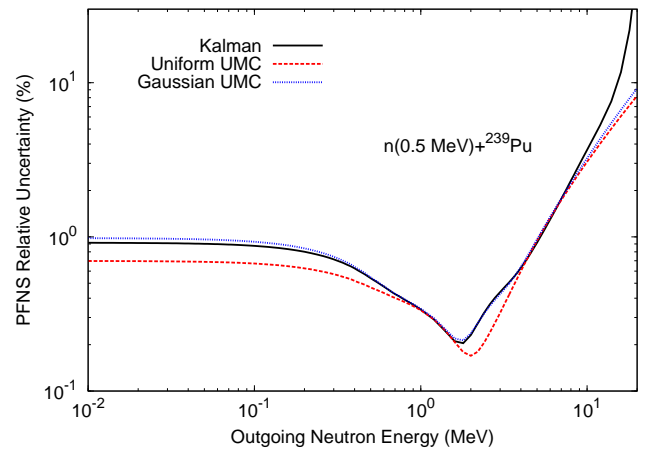

Figure 16: The PFNS relative uncertainty is plotted as a function of outgoing neutron energy, calculated using the Kalman filter and the UMC approach. The Gaussian UMC approach leads to very similar results as Kalman, except at the highest outgoing energies where the UMC results take into account the non-linearities of the Los Alamos model.

\subsection{Kalman Filter and Unified Monte Carlo}

We have used the Kalman filter Bayesian technique successfully in the past to quantify uncertainties and correlations for reaction cross sections [16] and PFNS [17]. In the present work, we have updated our previous Kalman filter code to work with LA model parameter systematics, as described above.

Another approach for quantifying uncertainties is the Unified Monte Carlo (UMC) method, as first discussed by Smith [18], and implemented on a simple toy model by Capote and Smith [19]. We have implemented this approach in a real evaluation situation for the first time [20]. In the UMC method, the probability distribution functions (PDFs) of the model input parameters are sampled through a Monte Carlo technique, and experimental data are used to minimize the $\chi^{2} / N$ between calculated and experimental PFNS values. We showed that the UMC and Kalman filter approaches lead to very similar mean values in most cases, but that the UMC method can fully account for non-linearities observed expected in the LA model, while the Kalman filter, at least in its first-order version, assumes linear relations between the model input parameters and the calculated PFNS. The example of $n(0.5 \mathrm{MeV})+{ }^{239} \mathrm{Pu}$ shown in Fig. 16 illustrates this point. 


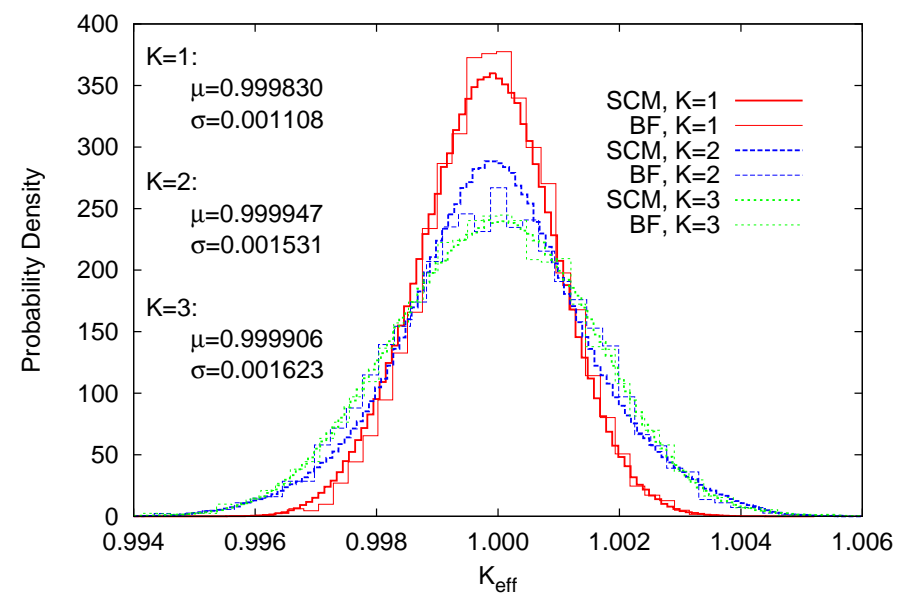

Figure 17: The probability density function of the effective multiplication factor, $k_{e f f}$, for the Jezebel fast critical assembly resulting from propagating the $\mathrm{n}+\mathrm{Pu}-239 \mathrm{PFNS}$ uncertainties through MCNP51.60 with a Gauss-Hermite quadrature set of order 8 with $8^{K}$ tensor product quadrature points. Note that the $k_{\text {eff }}$ results from the 10,000 directly sampled brute force realizations are also shown for each of the principal components with slightly thinner line thicknesses and wider histogram bins.

\subsection{Propagation of Uncertainties}

The uncertainties and correlations evaluated above for PFNS for suites of Pu and $\mathrm{U}$ isotopes have been propagated through fast neutron critical benchmark experiments to study their impact on integral data uncertainties. We have implemented the Polynomial Chaos Expansion (PCE) - Stochastic Collocation Method (SCM) to optimize these calculations. By performing a principal component decomposition of the PFNS covariance matrices, the size of the problem is significantly reduced and hinges on only 3 to 4 relevant eigenvalues. Normal and uniform random samplings of those eigenvalues are used in to fully characterize the final uncertainty in the final integral data being computed. Direct random sampling of the PFNS covariance matrices was used to benchmark the results obtained from the PCE-SCM calculations.

Such calculations were performed for the PFNS of thermal neutron-induced fission of U-235 and $\mathrm{Pu}-239$, and transport simulations were performed for the Godiva and Jezebel critical assemblies. The PCE-SCM results were in very good agreement with the direct sampling calculations, but required orders of magnitude less computational resources! An example comparing the results of the PCE-SCM and direct sampling techniques is shown in Fig. 17 for the probability distribution of the multiplication factor $k_{\text {eff }}$ in the case of the Jezebel fast critical assembly.

These very encouraging results are now being pursued in other transport simulations, as well as using the cross-isotope correlations that have been evaluated in the present work. 


\section{Conclusion}

This project addressed three important gaps identified in nuclear data evaluation libraries, and has produced significant results for each issue:

(1) Prompt fission neutron and $\gamma$-ray data can now be calculated for several actinides. Those data are not restricted to only the average spectrum and multiplicity, as what is currently stored in evaluated files, but contain distributions and correlations as well. In particular the neutron and $\gamma$-ray multiplicity distributions, multiplicity-dependent spectra, $\mathrm{n}-\mathrm{n}, \mathrm{n}-\gamma$ and $\gamma-\gamma$ correlations in angle and energy can now all be computed.

(2) Fully microscopic calculations of fission barriers have been performed for thorium, fermium, and plutonium isotopes. In addition, the inertia and level density along the fission paths can now be used in consistent fission cross section calculations using the R-matrix approach.

(3) New and unique uncertainty quantification tools have been developed and applied to the study of prompt fission neutron spectrum. For the first time, cross-isotope correlations have been evaluated. New uncertainty propagation techniques have been studied in transport simulations.

In addition to providing important new data to the U.S. Nuclear Energy programs, those results are opening new venues for computing other quantities of interest:

- Correlations (energy, angle) between prompt fission neutrons and $\gamma$ rays are being proposed for advanced detector simulations and for inclusion in the MCNP-6 transport code;

- Consistent fission cross section calculations across suites of isotopes using fully microscopic input data should lead to much improved predictive capabilities that are necessary to address some of the minor actinide needs for nuclear energy applications;

- Cross-isotope evaluations and uncertainty quantifications are being proposed for the U.S. ENDF/B-VII.1 library for the first time;

- New covariance matrix capabilities in transport simulations are being developed.

All results obtained as part of this project have been (or are in the process of being) published extensively in the scientific literature. 


\section{References}

[1] D.G.Madland and J.R.Nix, Nucl. Sci. Eng. 81, 213-271 (1982).

[2] S.Lemaire, P.Talou, T.Kawano, M.B.Chadwick, D.G.Madland, Phys. Rev. C72, 024601 (2005).

[3] S.Lemaire, P.Talou, T.Kawano, M.B.Chadwick, D.G.Madland, Phys. Rev. C73, 014602 (2006).

[4] P.Talou, T.Kawano, O.Bouland, J.E.Lynn, P.Möller, and M.B.Chadwick, Proc. of the International Conference on Nuclear Data for Science \& Technology ND2010, April 26-30, 2010, Jeju Island, Korea (2010). [LA-UR 10-03259]

[5] T. Kawano, P. Talou, M.B. Chadwick, and T. Watanabe, J. Nucl. Sci. Tech. 47, No. 5, 462 (2010).

[6] B. Becker, P. Talou, T. Kawano, Y. Danon, and I. Stetcu, accepted for publication in Phys. Rev. C.

[7] C.Romano, Y.Danon, R.Block, J.Thompson, E.Blain, and E.Bond, Phys. Rev. C81, 014607 (2010).

[8] P. Talou, B. Becker, T. Kawano, M.B. Chadwick, and Y. Danon, Phys. Rev. C83, 064612 (2011).

[9] H. Nifenecker, C. Signarbieux, M. Ribrag, J. Poitou, and J. Matuszek, Nucl. Phys. A189, 285 (1972).

[10] S.Bjornholm, J.E.Lynn. Rev. Mod. Phys., vol. 52, No. 4, 725-931 (1980).

[11] J.E.Lynn, A.C.Hayes. Phys. Rev. C67, 014607 (2003).

[12] M. Kortelainen, J. McDonnell, W. Nazarewicz, P.-G. Reinhard, J. Sarich, N. Schunck, M.V. Stoitsov and S.M. Wild, Phys. Rev. C85, 024304 (2012).

[13] A. Baran, J.A. Sheikh, J. Dobaczewski, W. Nazarewicz, and A. Staszczak, Phys. Rev. C84, 054321 (2011).

[14] J.A. Sheikh, W. Nazarewicz, and J.C. Pei, Phys. Rev. C80, 011302(R) (2009).

[15] A.Tudora, Annals of Nuclear Energy 36, 72-84 (2009).

[16] P. Talou, P.G. Young, T. Kawano, M.E. Rising, and M.B. Chadwick, Nucl. Data Sheets 112, No. 12, 3054 (2011).

[17] P. Talou, T. Kawano, D.G. Madland, A.C. Kahler, D.K. Parsons, M.C. White, R.C. Little, and M.B. Chadwick, Nucl. Sci. Eng. 166, 254-266 (2010).

[18] D.L. Smith, Proc. $8^{\text {th }}$ Int. Topical Meeting on Nucl. Applications and Util. of Accelerators, Pocatello, July 29-Aug.2, 2007, p.736 (2007).

[19] R. Capote and D.L. Smith, Nuclear Data Sheets 109, 2768 (2008).

[20] M.E. Rising, PhD Thesis, University of New Mexico, 2012. 


\section{Refereed Publications}

1. "Monte Carlo Hauser-Feshbach Predictions of Prompt Fission Gamma Rays - Application to $n_{\mathrm{th}}+{ }^{235} \mathrm{U}, n_{\mathrm{th}}+{ }^{239} \mathrm{Pu}$ and ${ }^{252} \mathrm{Cf}(\mathrm{sf}), "$ B. Becker, P. Talou, T. Kawano, Y. Danon, and I. Stetcu, Phys. Rev. C 87, 014617 (2013).

2. "Third minima in thorium and uranium isotopes in the self-consistent theory", J.D. McDonnell, W. Nazarewicz, and J.A. Sheikh, submitted to Phys. Rev. C (2013).

3. "Prompt Fission Neutron Spectrum Uncertainty Propagation using Polynomial Chaos Expansion," M.E. Rising, A.K. Prinja, P. Talou, accepted for publication in Nucl. Sci. Eng. [LAUR-12-25240]

4. "Evaluation and Uncertainty Quantification of Prompt Fission Neutron Spectra of U and Pu Isotopes," M.E. Rising, P. Talou, T. Kawano, and A.K.Prinja, accepted for publication in Nucl. Sci. Eng. [LA-UR-12-21035].

5. "Fission modes of mercury isotopes", M. Warda, A. Staszczak, and W. Nazarewicz; Phys. Rev. C 86, 024601 (2012).

6. "Quality Input for Microscopic Fission Theory", W. Nazarewicz, N. Schunck, and S. Wild, Stockpile Stewardship Quarterly, 2(1), May 2012, p. 6.

7. "Quadrupole collective inertia in nuclear fission: Cranking approximation", A. Baran, J. A. Sheikh, J. Dobaczewski, W. Nazarewicz, and A. Staszczak, Phys. Rev. C 84, 054321 (2011).

8. "Fission half lives of fermium isotopes within Skyrme Hartree-Fock-Bogoliubov theory", A. Baran, A. Staszczak, and W. Nazarewicz, Int. J. Mod. Phys. E 20, 557 (2011)

9. "Breaking of axial and reflection symmetries in spontaneous fission of fermium isotopes", A. Staszczak, A. Baran, and W. Nazarewicz, Int. J. Mod. Phys. E 20, 552 (2011)

10. "Surface symmetry energy of nuclear energy density functionals", N. Nikolov, N. Schunck, W. Nazarewicz, M. Bender, and J. Pei, Phys. Rev. C 83, 034305 (2011)

11. “Towards Predictive Theory of Fission”, W. Nazarewicz and J. McDonnell, Stewardship Science Academic Alliances Annual 2011 DOE/NA-0016, p.18 (2011)

12. "Nuclear energy density optimization: Large deformations", M. Kortelainen, J. McDonnell, W. Nazarewicz, P.-G. Reinhard, J. Sarich, N. Schunck, and M.V. Stoitsov. Phys. Rev. C 85, 024304 (2012).

13. "Advanced Monte Carlo Modeling of Prompt Fission Neutrons for Thermal and Fast NeutronInduced Fission Reaction on Pu-239," P. Talou, B. Becker, T. Kawano, M.B. Chadwick and Y. Danon, Phys. Rev. C 83, 064612 (2011).

14. "Augmented Lagrangian Method for Constrained Nuclear Density Functional Theory", A. Staszczak, M.Stoitsov, A. Baran, and W. Nazarewicz, Eur. Phys. J. A 46, 85 (2010).

15. "Fission barriers and neutron gas in compound superheavy nuclei", J.C. Pei, W. Nazarewicz, J.A. Sheikh, and A.K. Kerman, Nucl. Phys. A 834, 381c (2010). 


\section{Conference Proceedings}

1. "Monte Carlo Hauser-Fesbach Calculations of Prompt Fission Neutrons and Gamma Rays," P. Talou, T. Kawano, and I. Stetcu, to appear in Proc. of the $5^{\text {th }}$ Int. Conf. on Fission and Properties of Neutron-Rich Nuclei (ICFN5), Nov. 4-10, 2012, Sanibel Island, Florida (2012).

2. "Microscopic Description of Nuclear Fission: Fission Barrier Heights of Even-Even Actinides", J. McDonnell, N. Schunck, and W. Nazarewicz, , to appear in Proc. of the $5^{\text {th }}$ Int. Conf. on Fission and Properties of Neutron-Rich Nuclei (ICFN5), Nov. 4-10, 2012, Sanibel Island, Florida (2012).

3. "Excitation Energy Sorting Mechanisms in Fission," P. Talou, T. Kawano, I. Stetcu, and M.B. Chadwick, to appear in Proc. of the $13^{\text {th }}$ Int. Conf. on Nuclear Reaction Mechanisms, June 11-15, 2012, Varenna, Italy (2012).

4. "The Principal Component Analysis Method used with Polynomial Chaos Expansion to Propagate Uncertainties through Critical Transport Problems," M.E. Rising and A.K. Prinja, presented at the Advances in Reactor Physics Conference (PHYSOR 2012), Knoxville, Tennessee, April 15-20, 2012.

5. "Monte Carlo Hauser-Feshbach Modeling of Prompt Fission Neutrons and Gamma Rays," P. Talou, B. Becker, T. Kawano, and Y. Danon, Proc. of the Int. Conf. on Compound Nuclear Reactions CNR*11, Sep. 19-23, 2011, Prague, Czech Republic, EPJ Web of Conferences 21, 08003 (2012).

6. "Monte Carlo Simulation for Statistical Decay of Compound Nucleus," T. Kawano, P. Talou and M.B. Chadwick, Proc. of the Int. Conf. on Compound Nuclear Reactions CNR*11, Sep. 19-23, 2011, Prague, Czech Republic, EPJ Web of Conferences 21, 04001 (2012).

7. "Evaluation of Uncertainties for the Prompt Fission Neutron Spectrum and Average Neutron Multiplicity using the Los Alamos Model," M.E. Rising, P. Talou, and A.K. Prinja, presented at the American Nuclear Society: 2011 Annual Meeting, Hollywood, Florida, June 26-30, 2011.

8. "Application of Principal Component Analysis to Uncertainty Propagation in Radiation Transport," M.E. Rising, E.D. Fichtl, and A.K. Prinja, presented at the American Nuclear Society: 2011 Annual Meeting, Hollywood, Florida, June 26-30, 2011.

\section{Meetings}

1. "PFNS: Consistent Evaluations, UQ, and Propagation of Uncertainties," M.E. Rising, P. Talou, A.K. Prinja, T. Kawano, and M. White, presented at the Nuclear Data Week, Cross Section Evaluation Working Group, Brookhaven National Laboratory, November 5-9, 2012.

2. "The nuclear landscape: theoretical perspective", W. Nazarewicz, ICFN5, Sanibel Island, FL, Nov. 4-10, 2012.

3. "Microscopic Description of Fission Process", W. Nazarewicz, International Symposium ENSFN'12, University of Tokyo, Oct. 10-12, 2012. 
4. "Information content of a nuclear observable and systematic and statistical errors of nuclear DFT”, W. Nazarewicz, Inter-collaboration meeting, CEA Saclay, France, April 10-11, 2012.

5. “Theory, a Summary”, FUSHE 2012: ENSAR-ECOS Workshop on FUture SuperHeavy Element Strategy, W. Nazarewicz, Erbismühle-Weilrod, Germany, May 13-16, 2012.

6. "Systematic Uncertainty Propagation of Prompt Fission Neutron Spectra," M.E. Rising, P. Talou, T. Kawano, and A.K. Prinja, presented at the Nuclear Data Group Meeting, Los Alamos National Laboratory, April 4, 2012.

7. "Microscopic Description of Fission Process", W. Nazarewicz, 2012 SSAA Symposium, Washington DC, Feb. 22-23, 2012.

8. "Microscopic description of fission process within the Nuclear Density Functional Theory", W. Nazarewicz, Topical Meeting on "Theory of Nuclear Fission", GANIL, Caen, France. January 4-6, 2012.

9. "Prompt Fission Neutron Spectrum: Evaluation and Uncertainty Quantification for a Suite of Uranium Isotopes," M.E. Rising, P. Talou, T. Kawano, and A.K. Prinja, presented at the Nuclear Data Week, Cross Section Evaluation Working Group, Brookhaven National Laboratory, November 14-18, 2011.

10. "Fission Barriers of Compound Superheavy Nuclei", W. Nazarewicz, APS April Meeting 2010, Washington, DC, February 13-17, 2010.

11. "Microscopic Description of the Fission Process", W. Nazarewicz, 2010 SSAA Symposium, Washington, DC, Jan. 20-22, 2010.

12. "Improvements of Nuclear Data and its Uncertainties by Theoretical Modeling: Evaluation of Uncertainties for the Prompt Fission Neutron Spectrum and Average Multiplicity using the Los Alamos Model," M.E. Rising, A.K. Prinja, and P. Talou, presented at the AFCI-NEUP Collaboration Meeting, Santa Fe, USA, November 3, 2010. 